\title{
REPENSAR LOS RETORNOS A TRAVÉS DE LOS SISTEMAS DE MOVILIDAD EN Centroamérica. El caso de Nicaragua
}

\author{
Rethinking Returns through Mobility Systems in Central America. The Case of Nicaragua
}

\author{
Delphine Prunier
}

Resumen: En este artículo se propone una reflexión teórica sobre el concepto polisémico de retorno y la relación de los migrantes con su territorio de origen. A partir del caso de un municipio nicaragüense, se pretende analizar las dinámicas de movilidad y de circulación desde la perspectiva de los sistemas migratorios dispersos, considerando la diversidad de destinos y de temporalidades de desplazamiento dentro y fuera de la región centroamericana. La investigación busca demostrar que las lógicas de retorno abarcan realidades y experiencias múltiples dentro del proyecto migratorio. Con este objetivo, se discuten dos nociones sobre la articulación entre circulación, territorio y retorno: "reversibilidad" y "recurso espacial".

Palabras clave: retorno, migración, movilidad, circulación, migración de retorno.

Abstract: This article offers a theoretical reflection on the polysemic concept of return and the relationship of migrants with their home territory. Based on the case study of a Nicaraguan municipality, it analyzes the dynamics of mobility and circulation from the perspective of scattered migration systems, considering the diversity of both the destinations and the duration of movements that occur within and outside Central America. The article seeks to show that the migrants' understanding and interpretation of their return depend on multiple realities and experiences in Central America, . To this end, two concepts are discussed to enhance the debate on the link between circulation, territory and return: "reversibility" and "space resource".

Keywords: return, migration, mobility, circulation, resettlement.

Delphine Prunier. Doctora en Geografía por la Universidad París Diderot, Francia. Investigadora asociada en el Centro de Estudios Mexicanos y Centroamericanos (CEMCA), México. Artículo realizado en el marco del Programa de Becas Posdoctorales en la UNAM, Instituto de Investigaciones Sociales (IIS-UNAM), México. Temas de especialización: migración internacional, dinámicas de movilidad, ruralidad en México y Centroamérica. Correo electrónico: prunier. delphine@gmail.com.
Enviado a dictamen: 11 de abril de 2016. Aprobación: 1 de junio de 2016. Revisiones: 1. 


\section{Introducción}

$\amalg$ tema del retorno del migrante a su lugar de origen y sus potenciales impactos en el desarrollo es una preocupación cíclica tanto para las instituciones internacionales, como para los académicos especialistas en migración. ${ }^{1}$ Desde la crisis de 2008 en Europa y en Estados Unidos, el retorno de migrantes - por pérdida de empleo o debido a la deportaciónse ha vuelto un fenómeno masivo que nos obliga a considerarlo desde una nueva mirada, no solamente como casos marginales relacionados con un fracaso individual del proyecto migratorio, sino como parte de los mecanismos de movilidad integrados a la experiencia de los migrantes que se encuentran frente a contextos políticos y económicos que obstaculizan su capacidad de libre circulación. El fenómeno de los retornos masivos es de particular interés en el contexto México-Estados Unidos (Durand, 2004; Bhatt y Roberts, 2012; Masferrer y Roberts, 2012; Mestries 2013; Massey, Pren y Durand, 2009; Rivera, 2011), pero también en otras geografías, particularmente en el caso de la migración africana o magrebí en Europa, o de ecuatorianos y bolivianos en España, y de las llamadas políticas de "retorno voluntario" (Daum, 2002; Musette, 2007; Cassarino, 2007). Preocupan en particular las problemáticas de los impactos socioeconómicos de tales retornos en las localidades de origen, o de la reinserción en los ámbitos familiares y laborales, para estos migrantes que han estado muchos años fuera de su país.

En México, los análisis sobre migraciones internacionales se han centrado en la pareja migratoria México-Estados Unidos, en una situación que podemos calificar como "excepcional" a la luz de los múltiples y diversos patrones migratorios que se pueden observar en otras regiones del mundo. Entre estos dos grandes países se extiende una de las fronteras más grandes y simbólicamente más poderosas en términos de diferencial salarial, de representación de la fractura sur/ norte y, finalmente, de "desequilibrios y frustraciones" (Simon, 1995) tanto culturales como económicos. Los flujos migratorios mexicanos son unidireccionales, orientados principalmente hacia el vecino del norte.
Este punto de partida condiciona, en gran medida, la lectura de los procesos relacionados con el regreso de los migrantes a sus comunidades de origen. ${ }^{2}$ Frente a estos antecedentes muy marcados en la literatura mexicana, en este artículo se propone descentrar la mirada y poner a la luz "sistemas migratorios" distintos al mexicano para proponer una lectura renovada sobre el retorno de los migrantes. A través de la noción de sistema migratorio, buscamos subrayar los fenómenos de circularidad, "la relación, el intercambio, la circulación entre los diferentes lugares articulados por la migración internacional, practicados [léase utilizados] $]^{3}$ y conectados de varias formas por los migrantes y sus cercanos" (Simon, 2006: 6). Los sistemas migratorios son espacios constituidos, vividos, sensibles y representados: se considera que el migrante tiene capacidad de conectar los diferentes lugares del campo migratorio ${ }^{4}$ - a través de símbolos, de itinerarios, de intercambios, etcétera- $y$, de esta manera, de participar en la configuración de los territorios.

La región centroamericana, si bien se conoce como región de expulsión migratoria desde la cual nacen los flujos de tránsito que atraviesan México — con sus flujos paralelos relacionados al crimen y a la corrupción-, está comparativamente poco considerada como región de origen de los migrantes, y como espacio que genera dinámicas de movilidad regionales propias y procesos de transformación territorial a partir de la circulación de su fuerza de trabajo. Este texto se centra en particular en las dinámicas de movilidad, circulación y retorno que nacen en el territorio nicaragüense y se desarrollan a distintas escalas geográficas. El estudio de la migración en Nicaragua ha estado marcado por trabajos muy valiosos en los que se ha analizado la articulación entre regionalismo, actores sociales e identidad territorial (Morales, 2008), los sistemas migratorios tanto regionales como extrarregionales (Baumeister et al., 2008; Cortés Ramos, 2008; Barquero y Vargas, 2004; Castillo, 2010), y también los distintos megaproyectos y mercados laborales globales que atraen mano de obra migrante a escala regional (Morales, 2005; Morales et al., 2011), en particular hacia Costa Rica y El Salvador. Estas investigaciones dan cuenta de las múltiples 
formas de inserción de las sociedades centroamericanas dentro de las dinámicas transnacionales, de la estrecha interdependencia entre lo local y lo global, así como de la nueva división espacial del trabajo. Ahora bien, en este artículo proponemos una mirada que permita nuevas lecturas del retorno, al considerar la importancia de la relación de las mujeres y hombres migrantes con su territorio de origen —en estos casos en el medio ruralen el proceso de constitución de sus trayectorias de movilidad.

A partir del caso del municipio nicaragüense de Palacagüina, ${ }^{5}$ en el que hemos realizado diversas estancias de trabajo de campo entre 2009 y $2014,{ }^{6}$ damos cuenta de cómo los distintos mercados laborales en los que se insertan los migrantes - a escala tanto local como regional, nacional o internacional - organizan las oportunidades territoriales y cómo la gestión de la dispersión, de la circulación y del retorno forman parte de lógicas diferenciadas dependiendo del nivel de anclaje familiar.

Ante el retorno, nos encontramos frente a una noción polisémica, una palabra con diferentes usos para contextos muy diversos, desde casos de deportación, de retorno voluntario, de retorno de las segundas generaciones de migrantes, de retorno de jubilados o de visitas a la familia, hasta fenómenos de integración a mercados laborales temporales - agricultura o turismo, por ejemplo- o de circulación regional o interna. Así, múltiples acepciones de la idea de retorno se combinan o se contradicen, hasta cuestionar las maneras de leer el fenómeno migratorio y sus transformaciones recientes en el marco de las ciencias sociales. Partiendo de tal preocupación, en esta contribución se aspira a analizar y sistematizar esas diversas lógicas y a mostrar que corresponden a diferentes formas de integrar este momento de la trayectoria de movilidad dentro del proyecto migratorio. Después de una breve descripción de las dinámicas de dispersión, circulación y retorno presentes en el municipio de Palacagüina, proponemos en una segunda parte utilizar la noción de reversibilidad para dar visibilidad a las múltiples formas de retorno que se pueden combinar. En un tercer momento, planteamos que el retorno constituye en sí mismo un recurso espacial y que la construcción de la territorialidad está estrechamente articulada con el control de la movilidad - nivel de control que se define por la articulación entre la capacidad de agencia de los sujetos y las políticas migratorias ejercidas por autoridades nacionales-.

\section{La situación migratoria en Palacagüina: multipolaridad y dispersión de los flujos}

A escala nacional, los flujos nicaragüenses se dirigen esencialmente hacia dos destinos privilegiados (ver cuadro 1). Costa Rica es el primer destino de la migración originaria de Nicaragua. La circulación de la mano de obra nicaragüense en Costa Rica, de carácter histórico y tradicional, evolucionó en las décadas de 1970 y 1980 — marcadas por el conflicto armado - hacia patrones de instalación más durable. Hoy en día, la fuerza de trabajo, particularmente la de origen rural, se inserta en los mercados laborales de la agricultura de exportación, de la construcción, del servicio doméstico y de la seguridad privada (Baumeister et al., 2008; Morales, 2011). Se combinan modelos de migración temporal y prácticas de ida y vuelta según los calendarios de producción, con modelos de asentamiento de más larga duración con el grupo familiar, sin que las condiciones para lograr la regularización del estatus migratorio estén siempre reunidas. En segundo lugar, Estados Unidos representa el otro espacio atractivo para la mano de obra nicaragüense. Este campo migratorio se instaló durante el periodo de guerra civil y se ha venido estructurando con el tiempo, tomando un ritmo creciente a partir de la década de los años 2000. Esta migración - con una ligera mayoría masculina- está marcada por el carácter indocumentado de las estancias, lo que coloca a los migrantes en situaciones de gran vulnerabilidad social y de precariedad laboral.

A grandes rasgos, Palacagüina es una localidad representativa de la situación nacional. Cabe señalar, sin embargo, que la alternativa de migrar hacia uno de los países vecinos del $\mathrm{C}^{7}$ es particularmente común en este municipio, situado en las proximidades de la frontera con Honduras y a poca distancia de El Salvador. 
En estos espacios, los nicaragüenses pueden circular presentando simplemente su cedula de identidad, lo que facilita los desplazamientos temporales para empleos de tiempo estacional. La importancia de la migración regional y circular se refleja de manera más evidente al observar los datos que incluyen la totalidad de los viajes censados (ver cuadro 2): más de la cuarta parte de estos viajes se realizan hacia la región del C4 - contra el 8.4\% al considerar a los migrantes ausentes en el momento de la encuesta $-{ }^{8}$ y un $56.9 \%$ hacia Costa Rica - contra el 45\% en el momento de la encuesta-.

Finalmente, España es un destino emergente en el municipio de Palacagüina desde los años 2006 y 2007 - todavía muy minoritario a escala nacional-. Quienes integran este nuevo flujo migratorio son esencialmente mujeres que se dirigen hacia un mercado laboral de servicio doméstico y cuidado de niños y ancianos. En el momento de la encuesta, en 2009, sólo habíamos detectado de manera excepcional este fenómeno, pero en 2014 una nueva estancia de trabajo de campo nos permitió observar la importancia que había tomado esta dinámica, con un número creciente de mujeres que se habían ido a España, sobre todo a la región de Bilbao, País Vasco, donde se construyen las redes sociales que permiten la instalación y la inserción laboral.

Ahora bien, los datos cuantitativos recolectados permiten también dar cuenta de la frecuencia de los retornos al lugar de origen; nos enfocamos a observar las particularidades de cada campo migratorio en términos de temporalidades y movilidades espaciales en estos espacios dispersos. En la gráfica l puede observarse la frecuencia de los episodios de regreso al lugar de origen en función del primer lugar de destino en el itinerario de cada migrante (ver gráfica l).

Podemos constatar que la migración a Estados Unidos y a España se caracteriza por una posibilidad muy restringida de retorno ya que el costo humano y económico, asociado con el estatus migratorio generalmente irregular, no permite a los migrantes, en la gran mayoría de los casos, integrar con regularidad una etapa de regreso dentro de su trayectoria migratoria. $\mathrm{Al}$ contrario, los migrantes que se han dirigido hacia los mercados laborales regionales de los países vecinos -Costa Rica, El Salvador, Honduras y Guatemalatienen prácticas de circulación mucho más intensas, con un 60\% de casos en los que el migrante ha viajado y retornado por lo menos dos veces. En general, estos retornos frecuentes y regulares comienzan a ocurrir desde el inicio de la experiencia migratoria ya que, como veremos a continuación, se articulan con una cierta organización social y territorial de mercados laborales específicos.

\section{La noción de reversibilidad. Una perspectiva para dar visibilidad a las múltiples formas de retorno}

Para presentar la diversidad de las trayectorias migratorias que se pueden dibujar desde el espacio de origen y los diferentes marcos dentro de los cuales puede ocurrir el "evento" del retorno, proponemos apoyarnos en la noción de "reversibilidad" de la migración tal como la expusieron a finales de los años ochenta H. Domenach y M. Picouet (1987;1989), quienes invitan a "abordar las diversas formas de la migración bajo el ángulo de su (no)permanencia relativa, y más precisamente usando como nuevo criterio dominante el carácter de 'reversibilidad' eventual de la migración" (1987: 472). Nos apropiamos aquí de la tipología sugerida por estos autores para exponer los diferentes escenarios migratorios que se presentan en el estudio de caso nicaragüense, en términos de orientación de los flujos, inserción laboral, modalidades de circulación, condiciones de retorno y diferenciaciones de género. Bajo el prisma de la reversibilidad, podemos "situar" el retorno tanto temporal como espacialmente: se trata de una etapa de la trayectoria de movilidad que cobra sentido en el marco de una cierta práctica de los campos migratorios, con sus particulares ritmos y construcciones territoriales.

Los "flujos irreversibles" suponen abandonar la residencia-base: el desplazamiento y el cambio de espacio de vida se producen sin incluir en el sistema de reproducción familiar el espacio considerado como "de origen". Esta situación puede pasar en caso de migración forzada - conflicto, catástrofe natural, etcétera-, pero 
también de manera voluntaria - ruptura con el medio familiar, social, profesional o cultural de origen-. Nuestro trabajo de campo nos permite reportar estos fenómenos, tanto en dirección hacia países vecinos como hacia lugares más lejanos, pero son muy minoritarios en el corpus de nuestro análisis: al haber privilegiado un acercamiento metodológico desde las relaciones tejidas en la distancia con el lugar de origen y la parte de la familia que se queda, se han documentado muy poco estos casos de ruptura o de migración en donde el retorno no forma parte de las proyecciones - tanto del propio migrante como de sus familiares, de forma concreta o idealizada- Nos parece, sin embargo, importante mencionarlo como parte de la realidad migratoria nicaragüense, ya que la situación de guerra civil en los años ochenta del siglo pasado y el contexto de extrema pobreza y marginalización socioeconómica que se vive desde las tres últimas décadas ha provocado salidas definitivas sin intención o posibilidad de regresar al terruño. ${ }^{9}$

Por el contrario, los "flujos reversibles" hacen referencia a una residencia-base determinada. El punto de partida siempre es el mismo, pero los lugares de destino varían. Se diferencian tres tipos de flujos reversibles cuyas características se señalan a continuación.

\section{Los flujos reversibles de larga duración}

Se caracterizan por una situación de desigualdad económica muy pronunciada entre dos espacios. El retorno forma parte del proyecto migratorio y la conservación de relaciones transnacionales - afectivas, materiales o financieras - con el grupo de origen sirve para construir, asegurar o comprobar este objetivo (Cassarino, 2004). El regreso puede ocurrir al final de la etapa laboral, cuando las condiciones de jubilación se consideran más favorables en el país de origen que en el país de migración. Sin embargo, el endurecimiento de las políticas migratorias es un factor importante que llega a modificar de modo repentino la manera en la que los migrantes pueden imaginar o planificar su retorno. Por un lado, el número de deportaciones desde el interior del territorio estadounidense ha crecido de tal forma ${ }^{10}$ que el miedo a ser expulsado del territorio, separado de su familia y obligado de forma brusca a interrumpir su vida tanto social como laboral en migración, resulta cotidiano entre los hombres y mujeres migrantes que se encuentran en Estados Unidos, o bien en España. El retorno forzado se comporta como un elemento perturbador, un peligro, una traba que puede detener la experiencia migratoria y cancelar el carácter largo de la estancia. Por otro lado, la elevación de los costos - económicos y humanosdel tránsito y del cruce de fronteras ha forzado a los migrantes y sus familias a quedarse más tiempo en el lugar de destino, sin integrar etapas de retorno, ni siquiera de visita. En este contexto, la experiencia migratoria aparece como irreversible porque la eventualidad de un retorno - aunque sea temporal - se vuelve incompatible con el proyecto migratorio. En nuestro estudio, el escenario de la reversibilidad "de larga duración" corresponde con las trayectorias de los migrantes que se dirigen a Estados Unidos - en su mayoría hombres casados o solteros-o a España —en su mayoría madres solteras-. Para estos dos países de destino, el grado de reversibilidad de la migración es bajo porque el cierre de las fronteras, el costo del viaje y las situaciones de irregularidad de la estancia limitan las posibilidades de regreso y provocan, de alguna forma, que los migrantes se encuentren "atorados" en el país de destino, al ser la circulación un recurso inalcanzable para ellos. Esta situación es evaluada por el propio migrante y por su familia al momento de salir. Es un elemento clave de las estrategias que permiten definir el trayecto migratorio y evaluar los recursos de la movilidad correspondientes.

A estos factores se tiene que agregar el del ciclo de vida (Del Rey, 2004), cuyo papel es central en los mecanismos de proyección o de preparación del retorno. El lugar de formación de las uniones y del nacimiento de los hijos juega un rol mayor en la planificación del retorno o, al contrario, en la transformación del proyecto migratorio, que puede acompañarse de un aplazamiento sin fin del momento adecuado para regresar. Esto se observa notablemente cuando la persona que migra conforma una familia en migración, ${ }^{\text {ll }}$ o bien cuando sigue soltera, y las relaciones afectivas y económicas 
con la familia que se queda en el lugar de origen se desvanecen con el paso del tiempo. De ser lo contrario, la iniciativa de salir del pueblo se relaciona en general con la emergencia de nuevas necesidades conforme nacen y crecen los hijos, la voluntad de consolidar el capital material, la vivienda o algún negocio propio. El hombre o mujer que migra se convierte muchas veces, por medio del envío de remesas, en el único garante de los ingresos necesarios para la alimentación, educación y salud de los hijos, mientras las esposas o las abuelas están a cargo de su cuidado cotidiano. En cierta medida, la ausencia física permite la satisfacción de necesidades básicas y, en este sentido, la presencia simbólica. A cambio, el retorno significa el abandono de este estatus del migrante y un problema por lo que implica la ausencia de ingresos provenientes del exterior. Es preciso subrayar, sin embargo, que una de las diferencias mayores que existen entre hombres y mujeres migrantes tiene que ver con la definición de esta responsabilidad y del costo social que está ligado a las condiciones de movilidad. ${ }^{12}$

\section{Los flujos de reversibilidad constante}

Los flujos de tránsito son de duración variable y de periodicidad aleatoria. La frecuencia de los viajes es el elemento clave: puede variar en función del destino, de la dificultad para cruzar la o las fronteras, del sector laboral en el que se integra el migrante, o bien de la etapa en la que se encuentra dentro de su ciclo familiar y de su experiencia migratoria. Las dinámicas de movilidad nicaragüenses están ampliamente organizadas alrededor de este tipo de flujos, en los que la posibilidad de ir y venir entre origen y destino se articula con unas estancias de duración medianas o cortas. Podemos definirlas como tradicionales ya que se organizan, en gran parte, con base en un espacio económico regional específico: en Costa Rica, país vecino de Nicaragua, el dinamismo de la economía, los niveles de salarios, la estabilidad de la situación política y la oferta de empleos son elementos que atraen a una parte importante de la población migrante nicaragüense. "Las estrategias habituales de explotación", como las califican Domenach y Picouet (1987: 477), son particularmente importantes en los sectores de la construcción, del turismo, de la agricultura de exportación, de la seguridad privada y del servicio doméstico.

Las prácticas de ir y venir entre los dos países se inscriben en una tradición migratoria antigua a nivel regional y se explican por dos factores principales. Por un lado, la relativa permeabilidad de la frontera da la posibilidad a los migrantes de cruzar para dirigirse al mercado laboral vecino, ${ }^{13}$ pero también de regresar a su pueblo sin correr el riesgo de no poder volver a salir. El costo del viaje y de la visa no representa un factor limitante y las redes familiares tejidas desde varias generaciones entre Nicaragua y Costa Rica acompañan una gran capacidad de circulación en este campo migratorio particularmente fluido. Cabe mencionar, además, que una gran parte de los migrantes nicaragüenses en Costa Rica dispone de un estatus migratorio regular y estable, además de una experiencia larga de circulación en un "espacio de vida" (Courgeau, 1975; Cortes, 1998) compartido entre los dos países, entre dos ámbitos familiares. Por otro lado, las temporalidades propias de ciertos mercados laborales en Costa Rica son particularmente propicias a la circulación de la mano de obra. En efecto, los sectores de la agricultura de exportación — café, caña, piña, cítricos, etcétera- y de la construcción ofrecen empleos temporales, y la mano de obra masculina migrante organiza sus trayectorias, sus tiempos de estancia y sus momentos de regreso al pueblo, en función de la estacionalidad de la producción, permitiéndoles, en muchos casos, combinar la captación de ingresos en migración con el mantenimiento de una actividad agrícola o no agrícola en el espacio de origen. La reversibilidad se renueva constantemente y se traduce en estos casos por "una sucesión de ausencias, [...] por el paso repetitivo del estatus de 'presente' al estatus de 'ausente"' (Domenach y Picouet, 1987: 478). Las mujeres migrantes, trabajadoras domésticas, empleadas del sector terciario o amas de casa, consideran menos este tipo de calendario productivo para organizar su circulación: suelen regresar al pueblo para visitar a la familia o para la renovación de la visa, sin ejercer en el lugar de origen ninguna actividad asalariada. 


\section{Los flujos de reversibilidad esporádica}

De acuerdo con Domenach y Picouet, son flujos "organizados en función de la distorsión que se produce entre la capacidad que tiene el mercado de trabajo local, muchas veces inadaptada, y el carácter ocasional del fuerte crecimiento de la demanda de mano de obra" (1987: 478). Estos diversos flujos producen, en realidad, una simple extensión del mercado laboral local, empujando sus límites geográficos, en nuestro caso, hacia espacios vecinos como El Salvador, Honduras, Guatemala, o más recientemente Panamá. El objetivo de los migrantes es básicamente ampliar sus posibilidades de conseguir trabajo, aunque el diferencial en términos de nivel salarial no es grande. Se dirigen, por ejemplo, a la zona del Golfo de Fonseca, donde se realizan obras en el puerto o en carreteras, a zonas rurales de Honduras para realizar actividades de potrero, o bien a Guatemala para emprender pequeños negocios a escala regional. El área de acción es limitada, y los migrantes cambian de dirección justamente de acuerdo con las ofertas de empleo que surgen para temporalidades cortas, de manera fluctuante e imprevisible. Se trata en su gran mayoría de hombres, ya que los sectores productivos que corresponden con estas temporalidades de contratación demandan mano de obra masculina. Este tipo de ritmo de movilidad se caracteriza sobre todo por la fuerte repetición de los movimientos, por periodos de ausencia bastante cortos y por la alta frecuencia de las etapas de retorno. La estructuración del campo migratorio por los mercados laborales regionales influye en la reversibilidad tanto de los flujos mismos, como de los retornos: el lugar de origen en el medio rural se vuelve un espacio clave para el repliegue, la seguridad socioeconómica y la movilización de recursos territoriales o familiares anclados en el pueblo, en los periodos de alta vulnerabilidad para los migrantes en situación de desempleo.

Estos dos últimos tipos de reversibilidad de la migración - constante y esporádica - revisten modalidades temporales y espaciales particularmente características de las migraciones sur-sur: los ritmos y trayectorias de movilidad se adaptan a la formación de mercados laborales regionales y a la constitución de enclaves territoriales de tipo transnacional, cuyo dinamismo es totalmente dependiente de una fuerza de trabajo flexible y móvil, en cortas distancias y duraciones.

Considerando el contexto que nos ofrece el caso nicaragüense, nos parece importante discutir la noción de retorno y vincularla al análisis de los mecanismos de circulación y multiterritorialidad, a la manera que lo hace la geografía social francesa. Desde esta perspectiva, nos interesa realizar una lectura del retorno considerando el "anclaje familiar" de los migrantes en su lugar de origen y la manera como gestionan sus recursos espaciales.

\section{Experiencias de retorno múltiples, medio rural de origen y gestión de los recursos espaciales}

\section{Los distintos modelos de reversibilidad se combinan y se administran en el âmbito familiar rural}

A la par de un análisis de las dinámicas de movilidad, circulación y reversibilidad de los itinerarios de los migrantes, resulta muy útil considerar el retorno desde el lugar de origen, y en particular desde el anclaje espacial a cargo de la estructura familiar. En Palacagüina hemos observado cómo distintos flujos y tipos de reversibilidad migratoria se pueden combinar dentro de una misma familia. Los miembros activos de la familia rural llegan a conformar "espacios dispersos" (espaces éclatés en francés; Cortes, 1998) entre el lugar de origen y los diferentes lugares de migración: la organización social y productiva familiar se articula con la integración a los espacios del mercado laboral y con prácticas y ritmos de movilidad que pueden ser muy diversos para cada uno de los miembros móviles. Por ejemplo, podemos encontrar hogares en los que el padre va y viene de manera temporal entre Nicaragua y Costa Rica, mientras un hijo se encuentra desde hace varios años en Estados Unidos, o bien el caso de hogares en los que varios hijos o hijas son migrantes, unos a larga distancia - España o Estados Unidos-, otros circulando a escala regional y manteniendo una presencia temporal en el lugar de origen. 
En el caso de las movilidades que implican lógicas repetidas y frecuentes de circulación migratoria, la participación en la unidad productiva familiar en el lugar de origen es entrecortada, pero regular. Aquí, el retorno es un elemento constitutivo del mecanismo de subsistencia y de conservación del anclaje en el medio rural: la pluriactividad (Carton de Grammont, 2009), como mecanismo de diversificación de las actividades - tanto de los sectores como de los espacios en donde se realiza-, también constituye un recurso que se extiende desde el pueblo de origen hasta los espacios de migración. El retorno, que equivale a la presencia en el medio rural, tiene igual importancia para el funcionamiento de la economía familiar, que el acceso al empleo asalariado en migración. Dicho de otra manera, en estos casos de movilidad de corta duración, de diversificación espacial y sectorial de la actividad en los espacios cercanos, el retorno no representa el término de la experiencia migratoria —o de una de sus etapas - sino un momento de la vida activa plenamente integrado a la lógica de movilidad y de apropiación de los espacios, tanto para la producción de subsistencia como para el empleo asalariado en diversos espacios y a diversas escalas (Prunier, 2013).

En cambio, las migraciones de larga distancia y que se prolongan en el tiempo tienen otras consecuencias económicas, funcionales, profesionales y familiares en cuanto a la perspectiva del retorno. El deseo de regresar al pueblo - aunque sea al final de la vida laboral - se manifiesta generalmente al momento de irse, pero también a lo largo de los periodos de ausencia. Entre la familia y la persona involucrada en una dinámica de movilidad, la definición del momento apropiado para el retorno parece fijarse a partir de diversos procesos de evaluación de las oportunidades disponibles en diferentes puntos del espacio migratorio, incluyendo el lugar de origen.

De esta forma, es preciso subrayar la existencia de sistemas de movilidad muy diversos — según el tipo de fronteras que se franquean, las políticas migratorias en vigor, los mercados laborales involucrados, las formas y los ritmos de los movimientos-, pero también combinados: esta configuración dispersa, pero estructurada por medio de la unidad familiar, justifica haber centrado el trabajo de campo en el lugar de origen, espacio privilegiado para percibir y entender las dinámicas de adaptación espaciales y temporales, recursos principales de los migrantes y de sus familias. Como lo planteó $G$. Cortes en el caso de la dispersión migratoria en Bolivia:

[...] recurrir a la migración internacional necesita un uso de los recursos sociales y económicos a la altura de los riesgos, del costo y de las dificultades impuestas por una movilidad transfronteriza de larga distancia, [por esto] la familia en tanto que estructura social fundamental de la solidaridad y de la ayuda, permanece como figura central de las lógicas de movilidad (Cortes, 2011: 107).

\section{Dispersión, circulación migratoria y multiterritorialidad. ¿Es el retorno un recurso espacial?}

Partiendo de estos dispositivos complejos de dispersión familiar, hace falta ahora aportar elementos de respuesta a dos preguntas motores de esta reflexión: iqué es un recurso espacial? y ies el retorno un recurso espacial?

Para E. Ma Mung (2004), las configuraciones migratorias de tipo diaspórico se benefician de dos características principales: la "multipolaridad de la migración", es decir, la dispersión de los flujos, por un lado, y la "interpolaridad de las relaciones", esto es, la existencia de conexiones - simbólicas o reales - tanto con el lugar de origen como entre los distintos polos de la migración, por otro lado. Aunque la diáspora china estudiada por el autor se desarrolla sobre escalas espaciales e históricas difícilmente comparables con el caso nicaragüense, nos resulta muy sugerente poner de relieve la importancia de estos dos fenómenos para la comprensión del papel que juega el retorno dentro de las lógicas de gestión de los recursos espaciales. Pensando el retorno en términos de recursos y de oportunidades espaciales potencialmente utilizables en distintos puntos del sistema migratorio, podemos avanzar en la reflexión sobre la manera en la que la dispersión espacial, la movilidad, la pluriactividad, la multilocalización o la 
multipertenencia constituyen recursos, en el sentido de herramientas capaces de generar cambios o de favorecer la reproducción socioeconómica. La noción de recurso espacial se conecta con el estudio de las dinámicas de movilidad espacial, al considerar que los migrantes ejercen un uso diferenciado de los espacios y finalmente buscan beneficiarse de las diferencias territoriales, en especial en cuanto a las políticas migratorias restrictivas, los sectores laborales, los diferenciales de salarios, etcétera. La "intencionalidad de los actores" (Gumuchian y Pecqueur, 2007) junto con la capacidad de agencia y de toma de decisiones son factores centrales en esta interrelación entre territorio y recursos porque ambos elementos no están dados de por sí, sino que se fabrican socialmente. Finalmente, desplazarse, migrar, ir y venir, o regresar, constituyen recursos porque permiten apostar en diversos potenciales espaciales: la organización de los mercados laborales globales obliga a los trabajadores - y a fortiori a los migrantes - a adaptarse a la competitividad entre los territorios productivos. En este marco, los mecanismos de diversificación productiva y espacial conforman los pilares de las estrategias de sobrevivencia y de transformación de las familias rurales contemporáneas. Esto conduce a los migrantes y sus familias a una ampliación de sus espacios de vida y a una gestión siempre más compleja de los recursos espaciales, donde el retorno se convierte en uno de estos recursos y el medio rural en un espacio de base, repliegue y plataforma en contextos de alta precariedad para la fuerza de trabajo migrante.

Nociones como "circulación migratoria" o "territorios circulatorios" nos interpelan por sus capacidades de descifrar los sistemas migratorios en relación con las territorialidades involucradas. Los "territorios circulatorios" planteados por A. Tarrius $(1992,2000)$ ponen a la luz las prácticas de los actores migrantes - el "saber-migrar"- y la creación de normas entre el aquí y el allá. En este sentido, se hace una distinción entre una perspectiva centrada en la pareja "inmigración/ inserción" para privilegiar la pareja "migrante/territorio". De allí, emerge en la literatura francesa la noción de "circulación migratoria" - muy utilizada para explicar los mecanismos de circulación entre el Magreb y Europa (Dorai et al., 1998; Cortes y Faret, 2009; Audebert, 2008; Arab, 2009; Charef, 1999) - para evocar de forma dinámica las prácticas de ir y venir en términos de movilidad y de producción de los territorios. Al tomar en cuenta el carácter multisituado (Cortes y Pesche, 2013) de las construcciones territoriales y de las estrategias de uso de los espacios en contextos migratorios, invitamos a pensar el retorno como un recurso socioespacial que interviene en la construcción de los territorios de la movilidad y cuya accesibilidad es desigual — como todo recurso-

\section{Reflexiones finales}

Estudiar los retornos desde el lugar de origen, la movilidad y la reversibilidad permite valorar otras miradas y aportar a la discusión sobre la definición de este fenómeno. Nos parece que el retorno es parte de un sistema de movilidad que incluye distintas etapas, donde el lugar de origen forma parte de los recursos espaciales centrales para las familias rurales. Entre movilidad y arraigo, entre separación física y búsqueda de una estabilidad económica que permita cubrir las necesidades básicas de la familia que se queda, las mujeres y hombres que migran deben gestionar estos recursos espaciales dentro de un sistema migratorio disperso. En este mecanismo de evaluación, las temporalidades y las prácticas espaciales de movilidad constituyen elementos centrales que están estrechamente articulados con la relación que se mantiene o que se busca mantener con el territorio rural de origen. Regresar al lugar de origen es un paso que tiene un significado en términos de movilidad social, pero también de anclaje. En este sentido, y como resultado de este estudio, consideramos que las condiciones de retorno - o de no retorno- permiten sobre todo visualizar la relación que la persona migrante construye en torno a las distintas territorialidades y recursos espaciales que constituyen su campo migratorio. Así, mientras una parte de los migrantes se desplaza en los territorios circulatorios en movimientos de intensa reversibilidad — cuando las distancias y las 
condiciones de regularidad migratoria lo permitenpara reforzar el anclaje, y mantienen un pie en el tejido socioeconómico rural, otros migrantes emprenden itinerarios de migración que corresponden con una tensión espacial provocada por la larga distancia, las políticas de fronterización y control estatal, la imposibilidad de circular y el dilema de la integración.

Este estudio de caso nicaragüense nos ubica frente a un sistema migratorio pluridireccional, muy propicio para una discusión sobre los conceptos que permiten salir de una visión dual de la migración: se trata de un proceso que no se puede reducir a las problemáticas de la salida de los emigrantes o de la integración/ exclusión de los inmigrantes en sus lugares de destino. Al privilegiar la noción de movilidad sobre la de migración, se pretende poner el énfasis en la fluidez, la flexibilidad, la articulación de las escalas y la relación espacial y temporal con los territorios recorridos. En este sentido, la discusión sobre la relación entre movilidad y territorialidades nos parece muy estimulante: ¿cómo considerar la supuesta pérdida de importancia del territorio - "el fin de los territorios"- (Badie, 1995) en el mundo globalizado, donde las nuevas formas económicas, políticas y culturales de construcción de las relaciones sociales se basan mucho más en dinámicas de redes? ¿En qué medida, y sobre todo para quién, la dinámicas trasnacionales y los movimientos migratorios, siempre más complejos, provocan fenómenos de desterritorialización, de reterritorialización o de multiterritorialización? (Haesbaert, 2013; Herner, 2009; Moraes, 2009). En el contexto de esta reflexión teórica sobre los retornos nos parece importante insistir en el carácter desigual del acceso al recurso espacial y poner el énfasis en las condiciones de precariedad en las que los migrantes nicaragüenses experimentan la movilidad, la circulación, el retorno y, más generalmente, la multiterritorialidad — es decir la "experiencia simultánea y/o sucesiva de diferentes territorios reconstruyendo constantemente el propio" (Haesbaert, 2013: 34-35) - . El territorio, en tanto espacio relacional, se define a través de las relaciones de poder que en él se ejercen. El control de los movimientos, de las redes y del tiempo representa un desafío mayor en el mundo globalizado.
La experiencia del retorno sitúa a los migrantes en el corazón de esta articulación, ya que movilizan los recursos espaciales de la circulación migratoria y del anclaje rural con el objetivo de alcanzar mayor control sobre territorialidades múltiples y de producir así dinámicas de reterritorialización. Sin embargo, en la gran mayoría de los casos, dos factores muy poderosos otorgan a la movilidad un sentido desterritorializador para estas poblaciones: por un lado, la precarización de las condiciones de trabajo y de integración a los mercados laborales regidos por altos niveles de flexibilidad y, por otro lado, las políticas migratorias que acentúan las restricciones a la circulación al mismo tiempo que fuerzan a las instalaciones durables.

\section{Notas}

' Agradezco particularmente a la Dra. Sara María Lara Flores, asesora de mi investigación posdoctoral, por su lectura cuidadosa y sus valiosos comentarios.

2 Los destinos nacionales, por ejemplo, implican temporalidades de movilidad y de circulación distintas. Aunque forman parte de la realidad migratoria de numerosas familias mexicanas, estas dinámicas están muy poco consideradas a la hora de estudiar y discutir el fenómeno del retorno al lugar de origen (véase Prunier, 2013 sobre la integración de diversos ritmos y condiciones de movilidad dentro de trayectorias individuales o familiares, con una comparación entre México y Nicaragua).

${ }^{3}$ Nota de la autora.

${ }^{4}$ Nos referimos al "campo migratorio" (Simon, 1981, 2006; De Tapia, 1995; Faret, 2003) como el espacio estructurado por los flujos migratorios, donde la movilidad de los trabajadores está acompañada por todo tipo de movilidades inducidas: capitales, referencias culturales, bienes de consumo, prácticas sociales, etcétera.

5 Palacagüina es un municipio rural del norte de Nicaragua, situado en el departamento de Madriz, a 50 kilómetros de la frontera con Honduras. Está ubicado en la carretera panamericana, lo que le proporciona una buena conexión con las ciudades regionales, y cuenta 
con alrededor de 15000 habitantes, según evaluaciones de 2012 por parte del Instituto Nacional de Información de Desarrollo de Nicaragua.

${ }^{6}$ La información recolectada en campo se basa en dos tipos de fuentes complementarias. Por un lado, se llevó a cabo una encuesta cuantitativa a inicios de 2009, en el marco del Programa ANR TRANSITER (Dinámicas transnacionales y recomposiciones territoriales, bajo la dirección de Laurent Faret, 2008-2012, SEDET, Université Paris Diderot), CASE-LASEMA (CNRS/EHESS). La encuesta comprende 275 hogares, que fueron seleccionados de manera aleatoria, siempre y cuando uno o varios miembros del hogar tuvieran experiencia migratoria actual o pasada. Las preguntas trataron esencialmente de las actividades productivas agrícolas y no agrícolas y de las trayectorias migratorias de cada persona con experiencia migratoria pasada o actual. Contamos entonces para este municipio con información de 493 individuos con experiencia migratoria, de los cuales 232 se encontraban fuera en el momento de la encuesta. Por otro lado, se realizaron 41 entrevistas a profundidad con migrantes de regreso, con familiares de migrantes y con responsables locales entre 2008 y 2010. En 2014, otra serie de entrevistas (9) nos permitió profundizar sobre el tema de las temporalidades, condiciones y percepciones del retorno.

${ }^{7}$ El C4 es un acuerdo de libre circulación entre El Salvador, Honduras, Guatemala y Nicaragua.

${ }^{8} \mathrm{Al}$ considerar los datos que corresponden a la totalidad de los viajes censados, es decir, dedicando una parte de la encuesta a trazar todas las etapas de la trayectoria de movilidad de cada persona con experiencia migratoria -fecha, periodo, empleo, país de destino, etcétera para cada viaje realizado - , podemos tomar la medida de la importancia relativa de cada país de destino, enfatizando el carácter circulatorio de la migración nicaragüense. En particular, esto nos da la posibilidad de complementar la información obtenida con el conteo "simple" de los migrantes ausentes, suerte de fotografía en un momento $\mathrm{T}$, y nos permite poner en relieve las dinámicas de movilidad temporal y de retorno frecuente a escala regional.

${ }^{9}$ Es preciso subrayar aquí que, en sentido estricto, la migración sólo es verdaderamente irreversible cuando el migrante muere en el lugar de destino. Son numerosos los casos de personas que vuelven luego de veinte o veinticinco años, aunque no habían mantenido lazos ni intercambios con una base familiar, social o económica en el lugar de origen. La definición de la trayectoria y la decisión de regresar o no revelan una voluntad muy propia e íntima que hace en todo caso muy delicado el uso de la categoría de migración definitiva $-\mathrm{O}$ irreversible-

10 Crecimiento más fuerte que las deportaciones registradas desde la zona fronteriza (Pombo, 2010).

${ }^{11}$ Véase el estudio de Alba et al. (2014) sobre racialización y asimilación, matrimonios mixtos y segundas generaciones ciudadanos norteamericanos.

${ }^{12}$ Las mujeres en particular tienen que lidiar con el dilema de la buena/mala madre, entre responsabilidad económica y responsabilidad emocional, lo que lleva el tema del retorno al ámbito de una culpabilidad de doble filo: ¿debe una buena madre renunciar a los ingresos de la migración para regresar cerca de sus hijos?, ila responsabilidad maternal -o más generalmente parental- implica la presencia cotidiana o la capacidad de asegurar salud y educación de los hijos, aunque las condiciones del mercado laboral internacional posicionen a ciertos padres o madres en calidad de ausentes? (Azakura, 2014; Durin, 2014) La cuestión de la paternidad transnacional es un tema muy marginal en los estudios sobre migración y género. Así, C. Catarino y M. Morokvasic alertan: "El costo social que resulta de la ausencia, del alejamiento físico, emocional sólo está calculado al femenino. La separación de los padres con sus hijos no se piensa como realmente problemática, sino bajo el ángulo del apoyo económico destinado a la progenitura: iel padre migrante mandará o no remesas para su hogar, y en qué cantidades?" (2005: 7).

${ }^{13}$ Si una parte de los migrantes cruza de manera irregular, la mayoría de los entrevistados dice haber viajado con pasaporte y visa, aunque en varios casos, al no realizar los trámites de renovación, los trabajadores migrantes prolongaron su estancia en Costa Rica de manera irregular. 


\section{Referencias bibliográficas}

Alba, Richard, Tomás R. Jiménez y Helen B. Marrow (2014). "Mexican Americans as a Paradigm for Contemporary Intra-Group Heterogeneity". En Ethnic and Racial Studies, 37(3): 446-466.

Arab, Chadia (2009). Les Ait Ayad: la circulation migratoire des marocains entre la France, l'Espagne et l'Italie. Rennes: Presses Universitaires de Rennes.

Audebert, Cédric (2008). "La genèse de la notion de circulation migratoire: Retour sur une approche innovante des migrations". En E-Migrinter, 1: 2-4.

Azakura, Hiroko (2014). Salir adelante: experiencias emocionales por la maternidad a distancia. México: CIESAS

Badie, Bertrand (1995). La fin des territoires: essai sur le désordre international et sur l'utilité sociale du respect. Paris: A. Fayard.

Barquero, Jorge y Juan Carlos Vargas (2004). La migración internacional en Costa Rica: estado actual y consecuencias. San José: Academia de Centroamérica.

Baumeister, Eduardo, Edgar Fernández y Guillermo Acuña (2008). Estudio sobre las migraciones regionales de los nicaragüenses. Guatemala: Editorial de Ciencias Sociales.

Bhatt, Wasudha y Bryan R. Roberts (2012). "'Forbidden Return': Return Migration in the Age of Restriction". En Journal of Immigrant \& Refugee Studies, 10(2): 162-183.

Carton de Grammont, Hubert (2009). “La desagrarización del campo mexicano”. En Convergencia, 16(50): 13-55.

Cassarino, Jean-Pierre (2004). "Theorising return migration: The Conceptual Approach to Return Migrants Revisited". En International Journal on Multicultural Societies, 6(2): 253-279.

Cassarino, Jean-Pierre (2007). Migrants de retour au Maghreb:Réintégrationet enjeux de développement. Florence: RSCAS/European University Institute.

Castillo, Manuel Ángel (2010). "Las migraciones centroamericanas al norte: ihacia un sistema migratorio regional?" En Sara María Lara Flores (coord.), Migraciones de trabajo y movilidad territorial. México: Cámara de Diputados/CONACYT/Miguel Ángel Porrúa.
Catarino, Christine y Mirjana Morokvasic (2005) "Femmes, genre, migration et mobilités". En Revue Européenne des Migrations Internationales, 21(1): 7-27.

Charef, Mohammed (1999). La circulation migratoire marocaine: un pont entre deux rives. Rabat: Sud Contact.

Cortes, Geneviève (1998). "Migrations, systèmes de mobilité, espaces de vie: à la recherche de modèles". En Espace Géographique, 27(3): 265-275.

Cortes, Geneviève (2011). "La fabrique de la famille transnationale. Approche diachronique des espaces migratoires et de la dispersion des familles rurales boliviennes". En Autrepart, 57-58: 95-110.

Cortes, Geneviève y Denis Pesche (2013). "Territoire multisitué”. En L'Espace Géographique, 42(4): 289-292.

Cortes, Geneviève y Laurent Faret (2009). Les circulations transnationales: lire les turbulences migratoires contemporaines. Paris: A. Colin.

Cortés Ramos, Alberto (2008). "Development and Migration Dynamics between Nicaragua and Costa Rica: a Long Term Perspective". Tesis de doctorado en Geografía, Loughborough University. Reino Unido.

Courgeau, Daniel (1975). "Le concept de migration". En Actes du IVè Colloque de démographie africaine. Institut National de la Statistique et de la Démographie, République de Haute Volta, pp. 27-33.

Daum, Christophe (2002). "Aides au 'retour volontaire' et reinsertion au Mali: un bilan critique". En Hommes et Migrations, 1239: 40-48.

De Tapia, Stéphane (1995). «Le champ migratoire turc et l'Europe». En Alain Jund, Paul Dumont y Stéphane de Tapia (coords.), Enjeux de l'immigration turque en Europe. Les Turcs en France et en Europe. Paris: l'Harmattan.

Del Rey, Alberto (2004). "Movilidad y longevidad en las dinámicas familiares multigeneracionales. Aplicación al medio rural del Sotavento veracruzano, México". Tesis de doctorado en Demografía, Universidad Autónoma de Barcelona, Centro de Estudios Demográficos. Barcelona.

Domenach, Hervé y Michel Picouet (1987). "Le caractère de réversibilité dans l'étude de la migration". En Population, 42(3): 469-483.

Domenach, Hervé y Michel Picouet (1989). "Typologies et réversibilité migratoire”. En Reginald Appleyard 
(dir.), L'incidence des migrations internationales sur les pays en développement. Paris: OCDE.

Dorai, Kamel, Marie-Antoinette Hily, Frantz Loyer y Emmanuel Ma Mung (1998). "Bilan des travaux sur la circulation migratoire”. Informe para la Dirección de Población y Migraciones (Francia). Francia: Ministère de la Solidarité et de l'Emploi/MIGRINTER.

Durand, Jorge (2004). "Ensayo teórico sobre la emigración de retorno: el principio del rendimiento decreciente". En Cuadernos Geográficos de la Universidad de Granada, 35: 103-116.

Durin, Séverine (2014). "Servicio doméstico de planta, embarazo y crianza. Dilemas y estrategias de las trabajadoras domésticas en Monterrey”. En Séverin Durin, María Eugenia de la O y Santiago Bastos (coords.), Trabajadoras en la sombra. Dimensiones del servicio doméstico latinoamericano. México: CIESAS.

Faret, Laurent (2003). Les territoires de la mobilité: migration et communautés transnationales entre le Mexique et les États-Unis. Paris: CNRS.

Gumuchian, Hervé y Bernard Pecqueur (2007). La ressource territoriale. Paris: Economica.

Haesbaert, Rogério (2013). "Del mito de la desterritorialización a la multiterritorialidad". En Cultura y Representaciones Sociales, 8(15): 9-42.

Herner, María Teresa (2009). "Territorio, desterritorialización y reterritorialización: un abordaje teórico desde la perspectiva de Deleuze y Guattari”. En Revista Huellas, 13: 158-171.

Ma Mung, Emmanuel (2004). "Dispersal as a resource”. En Diaspora: A Journal of Transnational Studies, 13(2): 211-225.

Masferrer, Claudia y Bryan R. Roberts (2012). "Going Back Home? Changing Demography and Geography of Mexican Return Migration". En Population Research and Policy Review, 31(4): 465-496.

Massey, Douglas S., Karen A. Pren y Jorge Durand (2009). "Nuevos escenarios de la migración MéxicoEstados Unidos: Las consecuencias de la guerra antiinmigrante". En Papeles de Población, 15(61): 101-128.

Mestries, Francis (2013). "Los migrantes de retorno ante un futuro incierto”. En Sociológica, 28(78): 171-212.

Moraes Mena, Natalia (2009). "Del mito de la desterritorialización a la multiterritorialidad: una revisión conceptual a partir de la migración uruguaya". En Áreas: Revista Internacional de Ciencias Sociales, 28: 61-70.

Morales Gamboa, Abelardo (2005). Migraciones y territorios pobres en la globalización: nuevas territorialidades de la exclusión social en América Central. San José: FLACSO Costa Rica.

Morales Gamboa, Abelardo (2008). "Migraciones, regionalismo y ciudadanía en Centroamérica". En Daniel Villafuerte Solís y María del Carmen García Aguilar (coords.), Migraciones en el sur de México y Centroamérica. México: Miguel Ángel Porrúa/ UNICACH.

Morales Gamboa, Abelardo (comp.) (2011). Migración de relevo. Territorios locales e integración regional en Centroamérica. San José: FLACSO/PRISMA.

Morales Gamboa, Abelardo, Susan Kandel, Xenia Ortiz, Oscar Diaz y Guillermo Acuña (2011). Trabajadores migrantes y megaproyectos en América Central. San Salvador: UCA El Salvador/PNUD.

Musette, Mohamed Saib (2007). "Migrant de retour en Algérie. Une nouvelle stratégie en perspective?" Informe MIREM, Robert Schuman Centre for Advanced Studies, European University Institute.

Paris Pombo, Dolores (2010). "Procesos de repatriación: Experiencias de las personas devueltas a México por las autoridades estadounidenses". Documento de trabajo, noviembre 2010. México: COLEF/Woodrow Wilson International Center for Scholars.

Prunier, Delphine (2013). "De nouvelles ruralités en Amérique centrale? Dynamiques de mobilité, ressources et organisations familiales". Tesis de doctorado en Geografía. Université Paris Diderot. Francia.

Rivera Sánchez, Liliana (2011). “QQuiénes son los retornados? Apuntes sobre el migrante retornado en México contemporáneo". En Bela Feldman-Bianco, Liliana Rivera Sánchez, Carolina Stefoni Espinoza y Marta Inés Villa Martínez (coords.), La construcción social del sujeto migrante en América Latina: prácticas, representaciones y categorías. Quito: FLACSO.

Simon, Gildas (1981). "Réflexions sur la notion de champ migratoire international”. En Hommes et Terres du Nord, numéro especial Actes du Colloque International 
Migrations Internes et Externes en Europe Occidentale, Lille, 16-18 de octubre de 1980, pp. 85-89.

Simon, Gildas (1995). Géodynamique des migrationsinternationales danslemonde. Paris: Preses Universitaires de France.

Simon, Gildas (2006). "Migrations, la spatialisation du regard". En Revue Européenne des Migrations Internationales, 22(2): 9-21.
Tarrius, Alain (1992). Les fourmis d'Europe: Migrants riches, migrants pauvres et nouvelles villes internationales. Paris: L'Harmattan.

Tarrius, Alain (2000). "Leer, describir, interpretar. Las circulaciones migratorias: conveniencia de la noción de territorio circulatorio. Los nuevos hábitos de la identidad". En Relaciones, 21(83): 37-66.

\section{Cuadro 1. Repartición de los flujos migratorios por país de destino} a nivel nacional y de la localidad de Palacagüina

\begin{tabular}{|c|c|c|c|c|}
\hline & \multicolumn{2}{|c|}{$\begin{array}{c}\text { Nicaragua } \\
5604452 \text { habitantes }\end{array}$} & \multicolumn{2}{|c|}{$\begin{array}{l}\text { Palacagüina } \\
12825 \text { habitantes }\end{array}$} \\
\hline & Cifra absoluta & $\begin{array}{l}\text { Porcentaje sobre total } \\
\text { de población emigrante }\end{array}$ & Cifra absoluta & $\begin{array}{l}\text { Porcentaje sobre total de } \\
\text { población fuera del país } \\
\text { censada en la encuesta }\end{array}$ \\
\hline Costa Rica & 373548 & 51.2 & 107 & 45 \\
\hline $\begin{array}{l}\text { Estados } \\
\text { Unidos }\end{array}$ & 242886 & $33 \cdot 3$ & 92 & 38.6 \\
\hline $\mathrm{C}_{4}$ & 18977 & 2.6 & 20 & 8.4 \\
\hline España & 11540 & 1.6 & 9 & 3.8 \\
\hline
\end{tabular}

Fuente: Nicaragua: Bilateral Migration Matrix, 2010; Banco Mundial. Palacagüina: Encuesta Transiter, 2009.

Cuadro 2. Distribución de los migrantes, datos absolutos y porcentajes según la zona de destino. Municipio de Palacagüina, enero de 2009

\begin{tabular}{lcccccc}
\hline & \multicolumn{3}{c}{ Migrantes ausentes en el momento de la encuesta } & \multicolumn{2}{c}{ Total de los viajes censados } \\
\cline { 2 - 7 } & Mujeres & Hombres & Total & $\begin{array}{c}\text { Porcentaje } \\
\text { (ambos sexos) }\end{array}$ & Total & $\begin{array}{c}\text { Porcentaje } \\
\text { (ambos sexos) }\end{array}$ \\
\hline Costa Rica & 37 & 70 & 107 & 45 & 710 & 56.9 \\
Estados Unidos & 28 & 64 & 92 & 38.6 & 168 & 13.5 \\
C4 & 5 & 15 & 20 & 8.4 & 324 & 26 \\
España & 7 & 2 & 9 & 3.8 & 11 & 0.8 \\
Otros destinos & 3 & 1 & 4 & 1.7 & 23 & 1.9 \\
TOTAL & 80 & 152 & 232 & 100 & 1236 & 100 \\
\hline
\end{tabular}

Fuente: elaboración propia. 
Gráfica 1. Frecuencia de los retornos según el primer lugar de destino del migrante. Municipio de Palacagüina, enero 2009

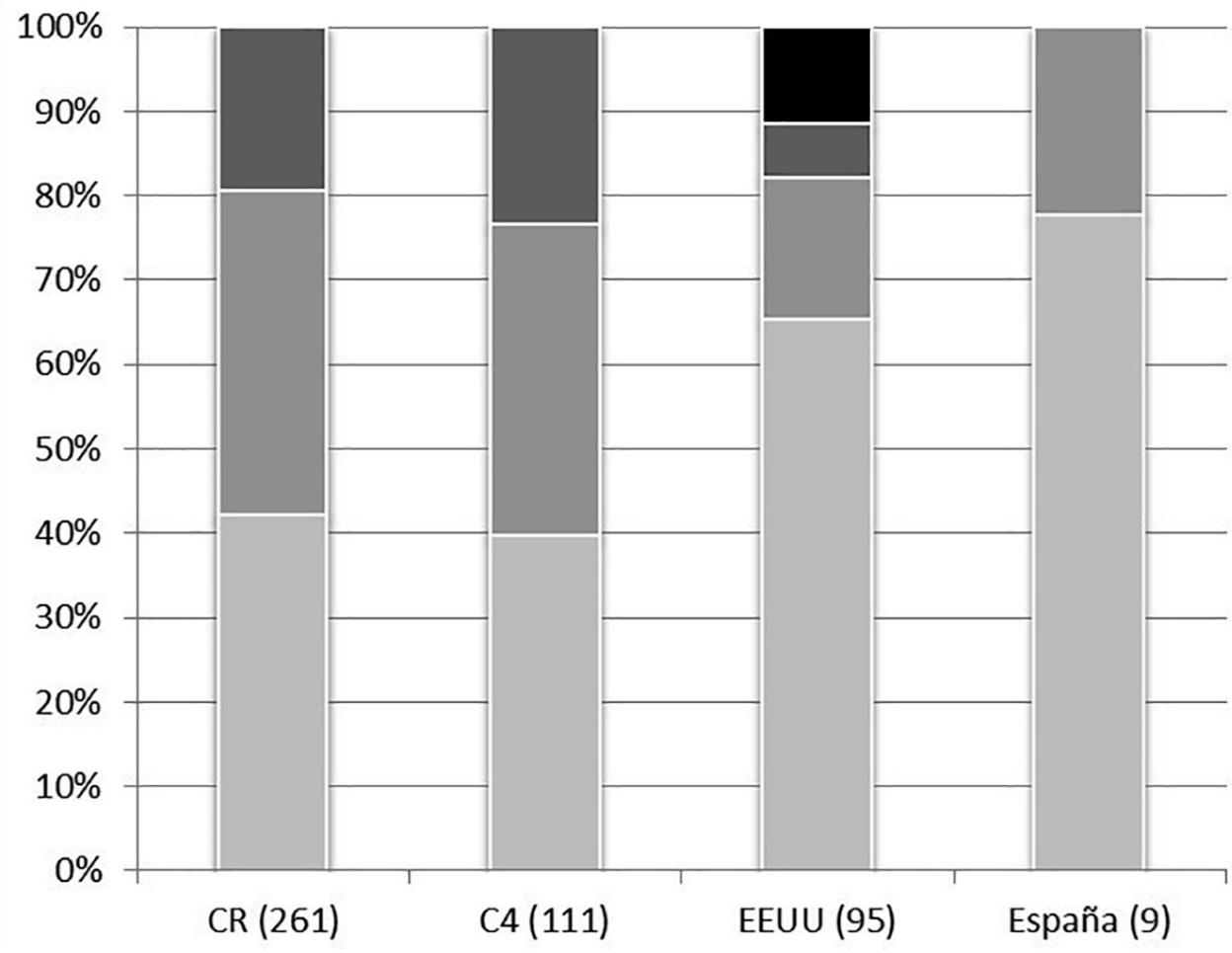

- Sin info

Más de 6 veces

De 2 a 5 veces

$1 \mathrm{vez}$

Fuente: Resultados de la encuesta TRANSITER, 2009. 